\title{
Maternal Suprachiasmatic Nuclei Are Necessary for Maternal Coordination of the Developing Circadian System
}

\author{
Steven M. Reppert and William J. Schwartz \\ Laboratory of Developmental Chronobiology and Neuroendocrine Research Laboratory, Children's and \\ Neurology Senvices, Massachusetts General Hospital and Harvard Medical School, Boston, Massachusetts 02114
}

\begin{abstract}
During late fetal and early neonatal life, the maternal circadian system coordinates the timing of a circadian clock in the hypothalamic suprachiasmatic nuclei (SCN) to the prevailing lightdark cycle. The role of the maternal SCN in the process of maternal coordination was investigated in rats. Complete lesions of the maternal SCN on day 7 of gestation disrupted rhythms of SCN glucose utilization in fetuses and pineal $N$-acetyltransferase activity in 10-d-old pups. This disruption was probably due to the desynchronization of individually oscillating fetal SCN, because individual pups born to and reared by SCN-lesioned dams under constant conditions exhibited normal circadian rhythms in drinking behavior. Cross-foster studies showed that the maternal circadian system can coordinate developing circadian rhythmicity during either the pre- or postnatal period. The results indicate that the maternal SCN are a necessary component of the mechanism of maternal coordination during both the pre- and postnatal periods.
\end{abstract}

In mammals, the suprachiasmatic nuclei (SCN) of the anterior hypothalamus contain a circadian pacemaker (biological clock) that controls the rhythmic expression of numerous physiological processes (Moore, 1983; Rusak and Zucker, 1979). The daily light-dark cycle entrains the SCN pacemaker to the $24 \mathrm{hr}$ day primarily through a monosynaptic retinohypothalamic pathway to the nuclei (Moore, 1981).

We recently used the ${ }^{14} \mathrm{C}$-labeled deoxyglucose (dG) autoradiographic method to examine the functional activity of the SCN clock during fetal development. The results of our $\mathrm{dG}$ experiments in rats show that the SCN are already oscillating during late prenatal life (Reppert and Schwartz, 1983, 1984); the fetal nuclei manifest a daily rhythm of glucose utilization (energy metabolism) with high activity during the day and low activity at night. We also showed that the fetal SCN are not directly synchronized by light that penetrates the maternal abdomen and uterus. Instead, it is the maternal circadian system that coordinates the timing (phase) of the fetal SCN to prevailing lighting conditions.

The maternal circadian system continues to coordinate the developing circadian system during the early postnatal period (Reppert, 1985). Maternal coordination wanes after the first week of life (Reppert et al., 1984; Takahashi and Deguchi, 1983) as the neonatal rat begins to respond to light directly through its own retinohypothalamic pathway (Reppert, 1984).

\footnotetext{
Received Nov. 7, 1985; revised Feb. 18, 1986; accepted Feb. 20, 1986.

We thank Robert Coleman, Matthew Morton, and Mark Banister for technical assistance, and Dr. L. Sokoloff and the Laboratory of Cerebral Metabolism for generous use of their densitometer apparatus. I his work was supported by PHS Grant HD 14427 and by Basic Research Grant 1-945 from the March of Dimes to S:M.R. W.J.S. is supported by NINCDS Teacher-Investigator Award K07 NS 00672. S.M.R. is an Established Investigator of the American Heart Association.

Correspondence should be addressed to Dr. Steven M. Reppert, Warren 10, Massachusetts General Hospital, Boston, MA 02114

Copyright (C) 1986 Society for Neuroscience $0270-6474 / 86 / 092724-06 \$ 02.00 / 0$
}

The present studies were designed to examine the role of the maternal SCN in the mechanism of maternal coordination. For these studies, lesions of the maternal nuclei were performed on day 7 of gestation, and the subsequent effects on 3 parameters of circadian development in the offspring were monitored; SCN glucose utilization was examined during prenatal life, while pineal $N$-acetyltransferase (NAT) activity and drinking behavior were monitored during the postnatal period.

\section{Materials and Methods}

\section{Animals}

Timed pregnant Sprague-Dawley rats (Zivic Miller Laboratories, Allison Park, PA) weighing 250-300 gm each at the time of insemination were housed by the supplier under diurnal lighting (LD: $12 \mathrm{hr}$ of light, with lights on from 0700 to $1900 \mathrm{hr}$ ) for several weeks prior to mating. Day 0 of gestation was designated as the day of sperm positivity. The animals were shipped to our facility within the first $2 \mathrm{~d}$ of gestation and housed singly in clear plastic cages; the cages in turn were contained within well-ventilated, lightproof environmental compartments. The LD cycle was automatically regulated; cool white light from fluorescent tubes delivered an intensity of 600 lux at the midcage level. During the dark portion of the lighting cycle, dim red light was provided by $20 \mathrm{~W}$ litho no. 2 fluorescent tubes (Chemical Products Co., North Warren, PA). These red lights also remained on constantly for animals maintained in constant darkness. Rat chow and water were always available, and the time of day of routine care was randomized.

\section{Surgical procedures}

All surgical procedures were performed under ether anesthesia. Dams and pups were blinded by bilateral orbital enucleation.

Bilateral lesions of the maternal SCN were made during the light period on day 7 of gestation by passing $4 \mathrm{~mA}$ of current for $10 \mathrm{sec}$ through bipolar platinum-iridium clectrodes insulated to within $0.5 \mathrm{~mm}$ of their tips $(A+7.5 \mathrm{~mm}$ anterior to ear bars, $\mathrm{L} \pm 0.3 \mathrm{~mm}$ from midline, $\mathrm{V}-9.5 \mathrm{~mm}$ from skull, incisor bar at the interaural line). For sham lesions, electrodes were placed just above the SCN $(A+7.5 \mathrm{~mm}$ anterior to ear bars, $\mathrm{L} \pm 0.3 \mathrm{~mm}$ from midline, $\mathrm{V}-8.5 \mathrm{~mm}$ from skull), and no current was passed. Sham and SCN-lesioned pregnant animals used in the dG studies were also outfitted with indwelling intra-atrial venous cannulae at the time of surgery (Reppert and Schwartz, 1983). The location and extent of lesions were verified by light-microscopic examination of cresyl violet-stained, 20- $\mu$ m-thick frozen coronal sections. Sections were cut at $60 \mu \mathrm{m}$ intervals.

\section{Drinking behavior}

Leads from a metal platform at the bottom of the cage and from the metal spout of a drinking tube were wired to a drinkometer relay (Lafayette Instruments, Lafayette, IN). A lick of the drinking tube registered a pen deflection on an event recorder (Esterline-Angus), so that the daily profiles of drinking behavior could be monitored.

\section{Determination of pineal NAT activity}

Pups were killed by decapitation. The pineal glands were then rapidly removed, placed in plastic microtubes on solid $\mathrm{CO}_{2}$ (within $30 \mathrm{sec}$ ), and stored at $-30^{\circ} \mathrm{C}$ until analysis. 
The assay of pineal NAT activity was a modification (Parfitt et al., 1975) of the method of Deguchi and Axelrod (1972). At the time of assay, each pineal gland was homogenized by sonication in $100 \mu \mathrm{l}$ sodium phosphate buffer $(0.1 \mathrm{M}, \mathrm{pH} 6.8)$ containing $1 \mu \mathrm{mol}$ of tryptamine $\mathrm{HCl}$ and $50 \mathrm{nmol}{ }^{14} \mathrm{C}$-acetyl coenzyme $\mathrm{A}$ (specific activity, $1 \mathrm{Ci} / \mathrm{mol}$ ). The reaction was terminated by extraction of the radiolabeled product $\left(N-{ }^{14} \mathrm{C}\right.$-acetyltryptamine) into $1 \mathrm{ml}$ chloroform. The chloroform was washed, and $500 \mu \mathrm{l}$ was then taken to dryness in a scintillation vial. Counting fluor was added, and the radioactivity in each sample was measured by liquid scintillation spectrometry. Data are expressed as $\mathrm{nmol}$ of product formed/gland/hr.

\section{$d G$ methodology}

At specified gestational ages and clock times (see below), pregnant rats were injected in the dark with $145 \mu \mathrm{Ci} / \mathrm{kg}$ dG (Amersham; specific activity, $60 \mathrm{Ci} / \mathrm{mol}$ ) through the indwelling venous catheter. The animals were kept in darkness until $\mathbf{4 5}$ min later, when each was killed by decapitation. The abdomen was then opened, and the fetuses were removed. Each fetal brain was carefully dissected out of the skull, frozen in cooled 2-methylbutane $\left(-30\right.$ to $\left.-40^{\circ} \mathrm{C}\right)$, and embedded with frozensection-mounting medium. Brains were stored at $-85^{\circ} \mathrm{C}$, and serial 20 $\mu \mathrm{m}$ coronal sections were then cut on a cryostat at $-20^{\circ} \mathrm{C}$ and dried on a hot plate. Autoradiographs were obtained as previously described (Reppert and Schwartz, 1984).

Optical densities (OD) of each fetal SCN were read on the Photoscan System P-1000 HS densitometer ( $50 \mu \mathrm{m}$ aperture) coupled to the computerized image-processing systcm of the Laboratory of Ccrebral Metabolism, National Institute of Mental Health. The OD of hypothalamus adjacent to SCN was also measured for each fetal brain in order to compare changes of OD of SCN between animals; thus, the data are expressed as relative OD (OD of SCN/OD of adjacent hypothalamus). For each brain, optical densities of the right and left SCN and the adjacent hypothalamus were measured over at least 3 adjacent sections.

\section{Statistical methods}

Analysis of the dG data was performed using the Mann-Whitney $U /$ Wilcoxon nonparametric test. Each population profile of NAT activity was analyzed for daily rhythmicity by analysis of variance. NAT population profiles were also subjected to sine-wave computer modeling; the resulting unweighted wave forms for each profile are depicted along with the individual data points in the figures. The computed sine waves were used solely for graphic clarity and not for statistical analysis. For all studies, statistical significance was set at $p<0.01$.

\section{Results}

\section{Maternal SCN lesions}

Drinking behavior was monitored in all dams before and after SCN lesions and was used to select the animals studied; more than $90 \%$ of dams with disrupted daily drinking profiles (assessed by visual inspection of the data) had histologically confirmed complete SCN lesions (Fig. 1). Dams with complete SCN lesions had no difficulty with parturition, and they nurtured their pups in a normal manner. A more detailed account of parturition in these animals will be reported elsewhere.

Rats with complete SCN lesions also had damage to the anterior, periventricular, and anterior ventromedial hypothalamic nuclei (Fig. 1). None of the lesions involved the paraventricular or supraoptic nuclei.

\section{Experiment 1: Effect of maternal SCN lesions on the rhythm} of metabolic activity in the fetal SCN

We first directly examined the effect of maternal SCN lesions on the function of the fetal clock by monitoring glucose utilization in the fetal nuclei. Sham and SCN-lesioned pregnant rats were maintained in LD until day 19 of gestation, when the animals were placed in constant darkness in preparation for the $\mathrm{dG}$ injections. Animals were then injected during either the subjective night (the $12 \mathrm{hr}$ period of LD when the lights would have been off) at $2300 \mathrm{hr}$ on day 20 of gestation, or the subjective day at $1100 \mathrm{hr}$ on day 21 of gestation. For the sham group, all fetal brains from 2 pregnant rats were examined at each of the 2 injection times. For the SCN-lesioned group, all fetal brains from 3 pregnant rats were examined at each of the 2 times.

In the fetuses from sham-operated dams, the SCN manifested a clear day-night rhythm of metabolic activity indistinguishable from that found in fetuses from unoperated animals (Fig. $2 \mathrm{~A}$; $p<0.01$ ); that is, virtually all fetal SCN were metabolically active and visible in the autoradiographs during subjective day, and they were all metabolically inactive and not visible during subjective night. In contrast, there was no rhythm of SCN metabolic activity in fetuses from SCN-lesioned dams (Fig. $2 A$ ). In this case, the mean values of SCN metabolic activity at both injection times were similar and intermediate between the normally high day and low night values. Visual inspection of these autoradiographs showed that the metabolic activity of the individual fetal SCN within each litter was quite varied and spanned the inactive (invisible) to active (visible) range (Fig. $2 B$ ), a condition never found within litters from either intact or shamoperated dams (see above). Thus, maternal SCN lesions on day 7 of gestation disrupt the normal rhythmic pattern of metabolic activity in the fetal SCN.

\section{Experiment 2: Effect of maternal SCN lesions on pineal NAT activity in 10-d-old pups}

We next examined the effect of maternal SCN lesions on the rhythm of pineal NAT activity in 10-d-old pups; the pineal NAT rhythm is one of the first circadian rhythms overtly expressed in rats (Reppert, 1982), and it accurately reflects circadian output from the developing SCN (Deguchi, 1982). Sham and SCN-lesioned pregnant rats were housed in LD throughout pregnancy. The dams were placed in darkness $2 \mathrm{~d}$ before birth, and the pups were born and reared in constant darkness. At 10 $\mathrm{d}$ of age, pups were killed in darkness every $2 \mathrm{hr}$ for a $24 \mathrm{hr}$ period; 4-6 pups of mixed sexes were randomly selected from different dams at each time point, and each experimental group consisted of 5-7 dams.

The population profile of pineal NAT activity for the pups born to sham-operated dams exhibited a significant daily rhythm ( $p<0.01$; Fig. 3 , upper panel). The rhythm was similar to that reported for pups born to unoperated dams and was characterized by high NAT values during the subjective night (Reppert et al., 1984). The population profile of pineal NAT activity for the pups born to SCN-lesioned dams, on the other hand, showed considerable scatter at all clock times, and there was no significant daily rhythm (Fig. 3, lower panel). The high NAT values of these pups are within the nighttime range of high values normally seen in pups born to intact dams, even though these values were lower than those found in the pups born to the sham-operated dams shown in the upper panel of Figure 3. Thus, maternal SCN lesions also disrupt a circadian rhythm normally expressed during the postnatal period.

\section{Experiment 3: Effect of maternal SCN lesions on pup drinking behavior}

Since it was not possible to determine from the dG and NAT experiments whether the developing SCN were still individually oscillating out of phase with one another after maternal SCN lesions, we examined the effect of such lesions on drinking behavior, a rhythmic parameter that could be monitored continuously in individual animals during the postnatal period. Sham and SCN-lesioned rats were housed in LD throughout pregnancy. The dams were placed in darkness $2 \mathrm{~d}$ before birth, and the pups were born and reared in constant darkness. On postnatal day 2 , all dams and pups were blinded in darkness with the aid of red light. On postnatal day 21 , the male pups were weaned and placed in individual cages, and drinking behavior was continuously monitored for the following 21 days. The phase of drinking offset at the time of weaning was estimated for each 

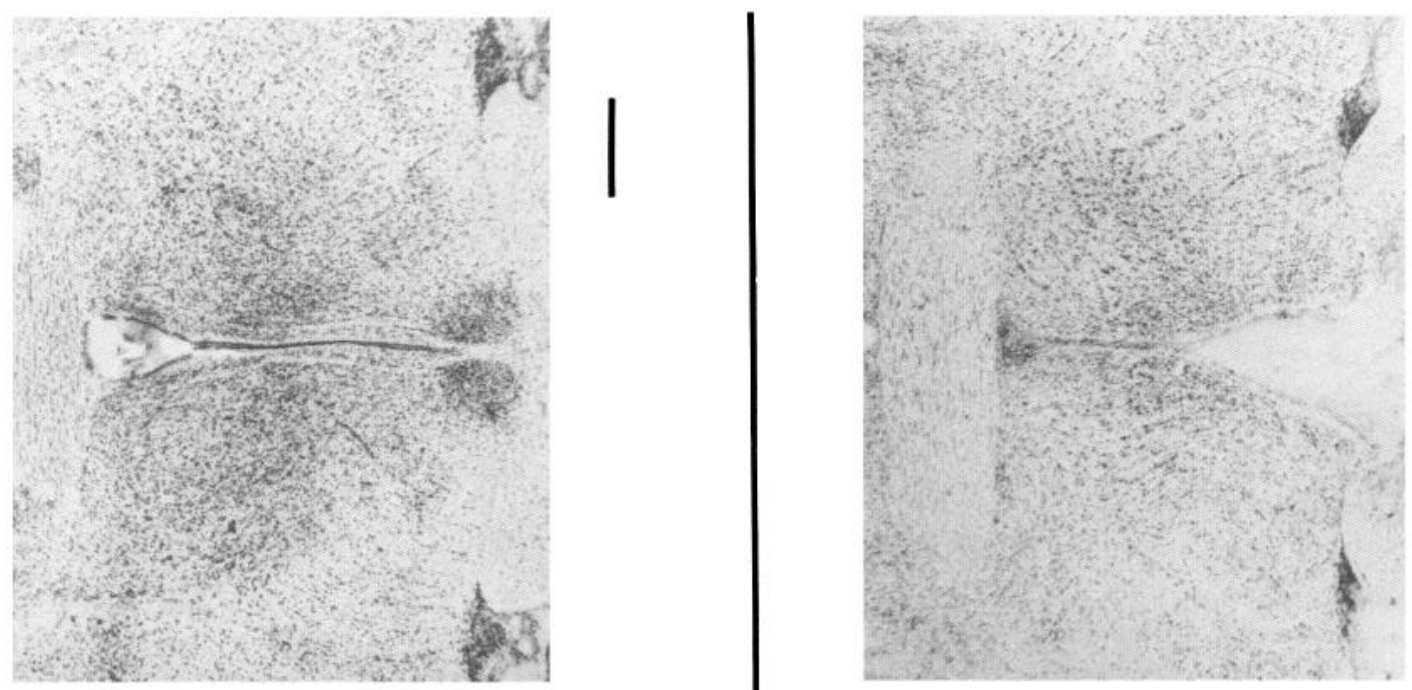

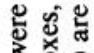

3.

ह

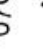
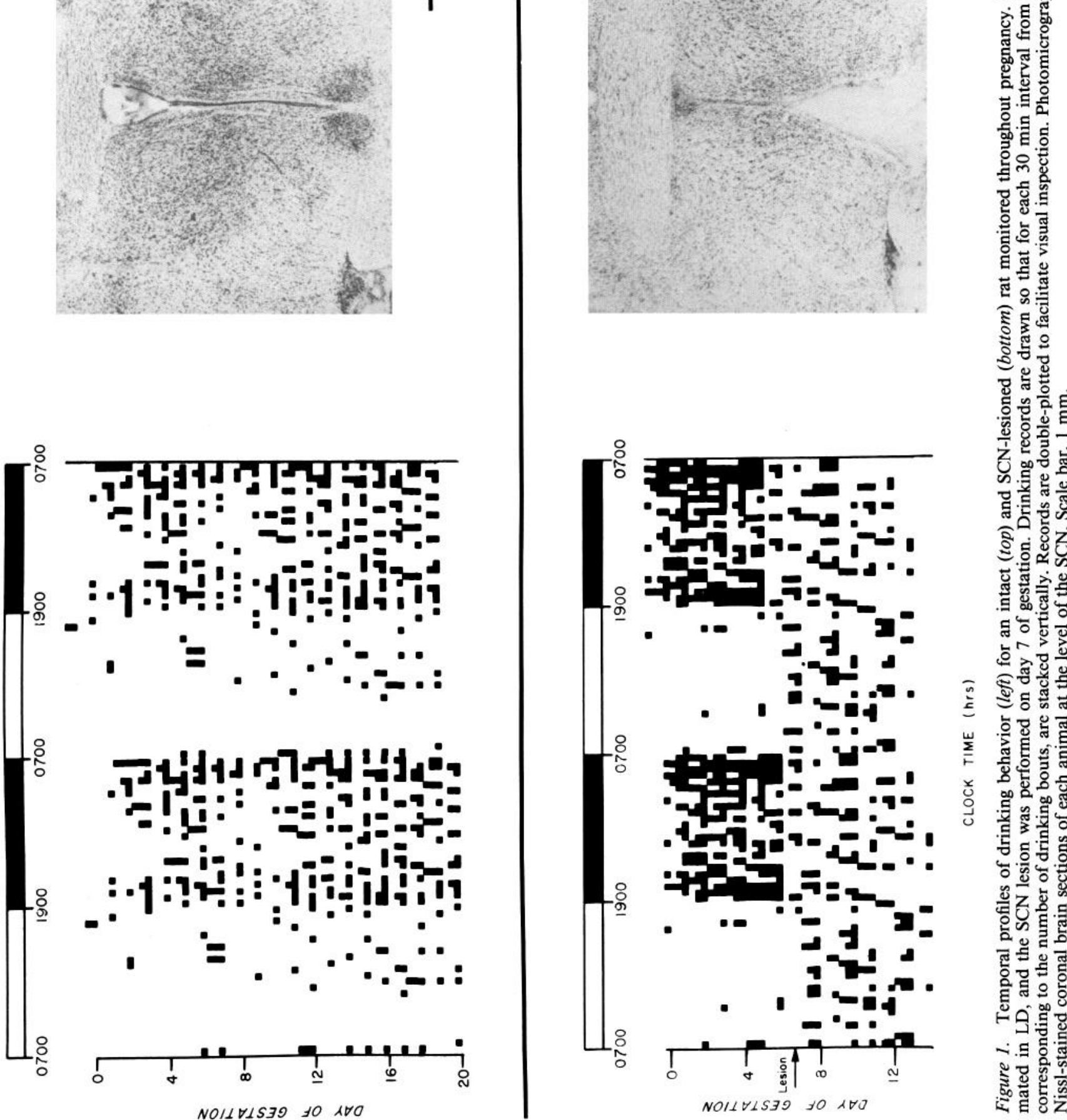
A.

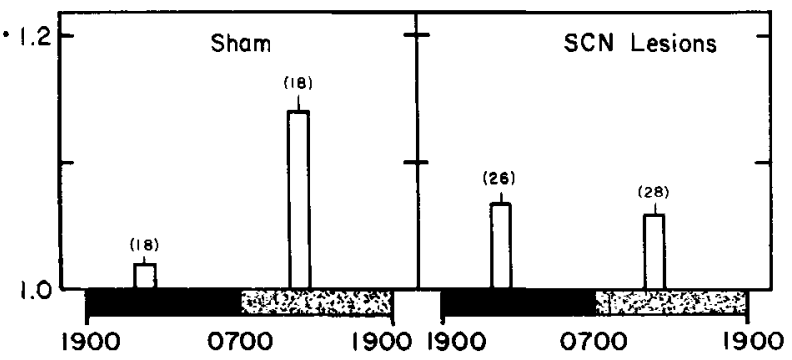

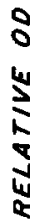

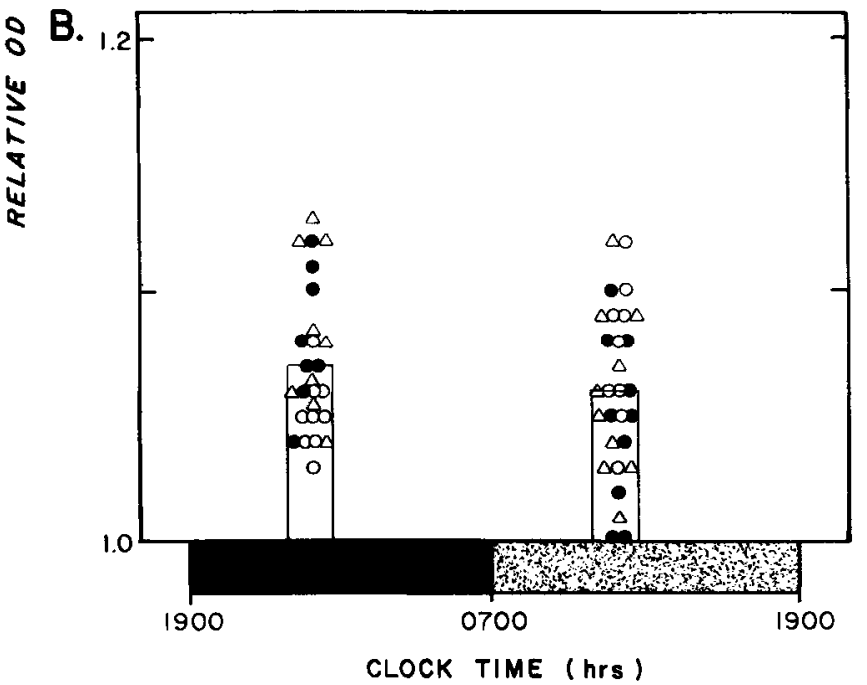

Figure 2. A, Day-night profiles of relative OD of fetal SCN from shamoperated dams (left) and from dams with histologically complete SCN lesions (right). For each injection time in the sham-operated group, all fetuses were studied from each of 2 pregnant rats. For each injection time in the SCN lesion group, all fetuses were studied from each of 3 pregnant rats. Vertical bars, mean \pm SEM; number of fetuses in parentheses. A significant day-night variation of metabolic activity was present in fetal SC.N from the sham-operated dams $(p<0.01)$ and absent in those from lesioned dams. $B$, The OD of each fetal SCN examined from mothers with SCN lesions. At each time point, the open circles represent the values of the first litter, closed circles the values of the second litter, triangles the values of the third litter. Stippled area of the lighting cycles represents subjective day, solid black bar subjective night.

animal by drawing an eye-fitted line through the daily offset times for the $21 \mathrm{~d}$ period (Pittendrigh and Daan, 1976).

All the pups from both groups manifested clear free-running rhythms of drinking behavior (Fig. 4). The mean free-running cycle length for both groups was similar: $24.46 \pm 0.02 \mathrm{hr}$ (mean \pm SEM, $n=9)$ for the sham group and $24.41 \pm 0.04 \mathrm{hr}(n=14)$ for the SCN-lesioned group. Thus, individual pups born to and reared by $\mathrm{SCN}$-lesioned dams generate normal free-running circadian rhythms during the postnatal period. The phases of drinking offsets at weaning for the animals born to and reared by the 2 sham-operated dams were very similar-range of only $3 \mathrm{hr}$ (Fig. 5). The phases of drinking offsets for the animals born to 2 of the 3 SCN-lesioned dams were more varied, spanning ranges of $7 \mathrm{hr}$ (triangles) and $12 \mathrm{hr}$ (open circles); the drinking profiles of 2 pups from the same litter that express offset times $12 \mathrm{hr}$ out of phase with each other are depicted in Figure 4. Similar phases of drinking offsets were found for the 3 pups born to the third SCN-lesioned dam (Fig. 5, closed circles).

Experiment 4: Relative influence of maternal coordination of the developing circadian system during the pre- and postnatal periods

Cross-foster studies were used to evaluate the relative potency of maternal entrainment during the pre- and postnatal periods.

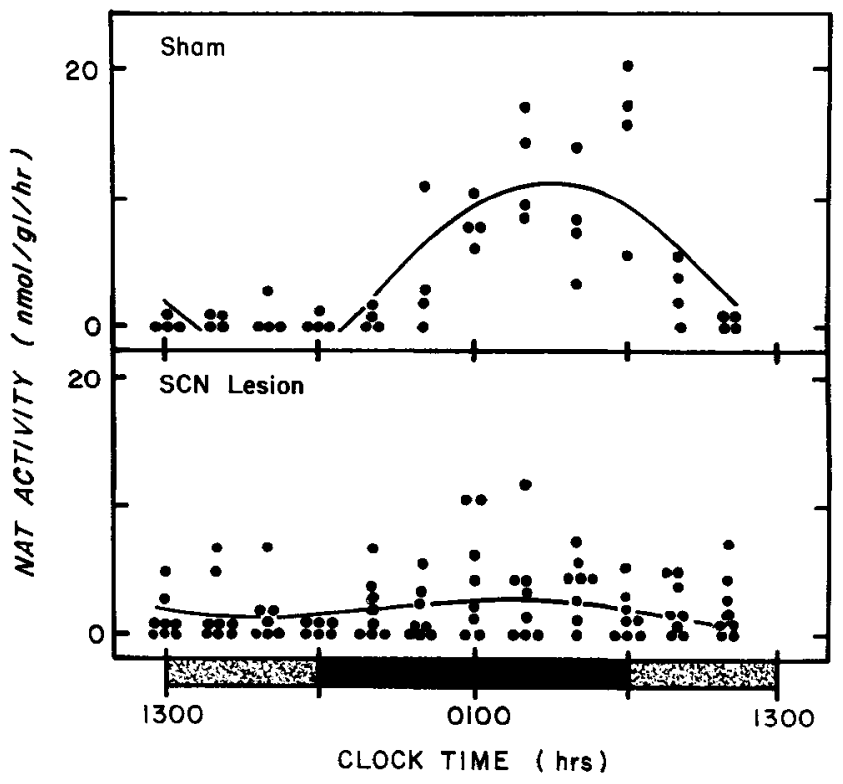

Figure 3. Daily profiles of NAT activity for 10-d-old pups born to sham-operated (top) or SCN-lesioned (bottom) dams. Dams were reared in LD during pregnancy, and surgery was performed on day 7 of gestation. Two days before birth, the dams were placed in constant darkness, and the pups were born and reared in constant darkness. Pups of both groups were killed in constant darkness over a $24 \mathrm{hr}$ period on postnatal day 10. Each data point is the NAT value of a single pup. Waveforms were derived from sine-wave analysis of the profiles. Stippled area of the lighting cycle represents subjective day.

Intact and SCN-lesioned pregnant rats were housed in diurnal lighting throughout pregnancy. The dams were placed in darkness $2 \mathrm{~d}$ before birth, and the pups were born and reared in constant darkness. Within $24 \mathrm{hr}$ after birth, the dams were exchanged so that pups born to intact dams were reared by SCNlesioned dams (designated Foster A), and the pups born to SCNlesioned dams were reared by intact dams (designated Foster B). At $10 \mathrm{~d}$ of age, pups of both groups were killed throughout a 24-hr period as described for experiment 2.

For both groups the population profiles of pup NAT activity exhibited significant daily rhythms $(p<0.01$; Fig. 6). The Foster A group had a rhythm that was similar to that found for pups born to and reared by intact or sham-operated dams. The population profile of the Foster B group appeared more scattered than the Foster A profile.

\section{Discussion}

Davis and Gorski (1983), using Syrian hamsters, were the first to show that ablation of the maternal SCN on day 7 of gestation disrupts the timing of the developing circadian system. These investigators monitored locomotor activity in individual pups reared under constant conditions. At the time of weaning, pups born to and reared by $\mathrm{SCN}$-lesioned dams expressed scattered phases of wheel-running behavior, while pups of sham-operated dams expressed similar phases.

The data presented in this paper show that the maternal SCN are also a necessary component of the mechanism for maternal coordination of the fetal circadian clock in rats. Rhythms of SCN metabolic activity in fetuses (experiment 1) and pineal NAT activity in 10-d-old pups (experiment 2) were both disrupted following maternal SCN lesions. Because the rhythms were monitored from a population of animals, their disruption could indicate either loss of rhythmicity or desynchronization of persistent rhythms among the individual animals within a litter. The latter interpretation is supported by the results of pup 
A.

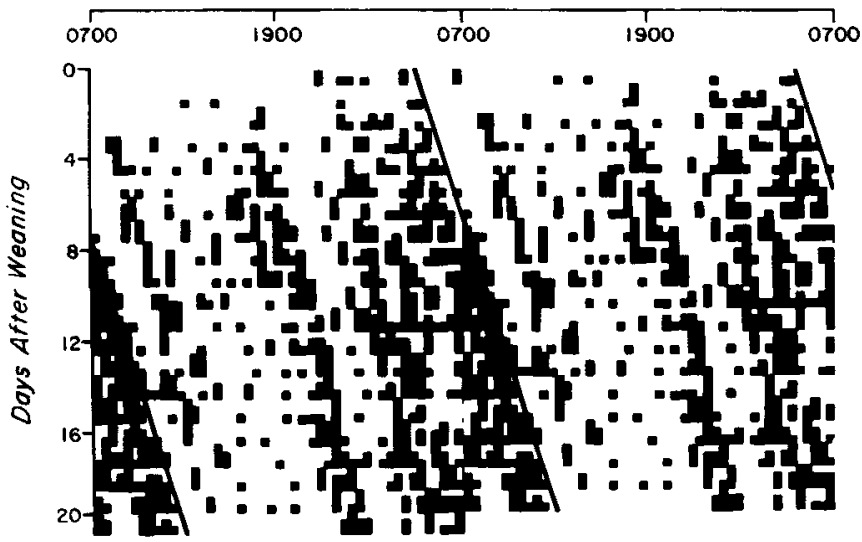

B.

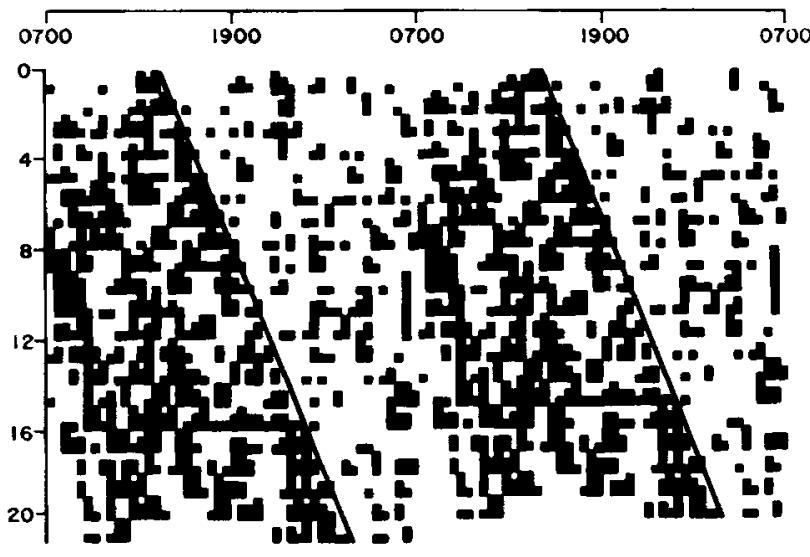

Clock Time (hours)

Figure 4. Temporal profiles of drinking behavior for 2 pups born to and reared by an SCN-lesioned dam. Dam was reared in LD throughout pregnancy, and lesioning was performed on day 7 of gestation. Two days before birth, the dam was placed in constant darkness, and the pups were born and reared in constant darkness. On postnatal day 2 , the dam and her pups were blinded. On postnatal day 21 , the pups were weaned and placed in individual cages; drinking behavior was monitored for the subsequent 21 days. Drinking records are drawn so that, for each 30 min interval, from 0 to 4 boxes, corresponding to the number of drinking bouts, are stacked vertically. An eye-fitted line is drawn through the daily offset times of drinking for the $21 \mathrm{~d}$ period. Records are double-plotted to facilitate visual inspection.

drinking behavior (experiment 3 ), which clearly show that the developing circadian system is oscillating and generating freerunning circadian rhythms in individual pups following prenatal maternal SCN lesions.

It is worth noting that at weaning the phases of drinking offsets within litters born to and reared by 2 of the SCN-lesioned dams were considerably more varied than those of pups born to shamoperated dams; phases ranged over 7 and $12 \mathrm{hr}$ for the litters of SCN-lesioned dams compared to $3 \mathrm{hr}$ for the litters of shamoperated dams. A random distribution of phases at weaning for pups born to SCN-lesioned dams would be expected only if the phases of SCN oscillation were random at their inception for fetuses within a litter and the individual fetal clocks expressed identical free-running period lengths. Thus, less-than-random phases of the SCN oscillation at inception and variation in individual free-running period lengths could account for the less than random variation found in the 2 litters from SCN-lesioned dams. The pups of the litter from the third SCN-lesioned dam expressed similar phases to each other at weaning but markedly different phases from pups of sham-operated dams (Fig. 5).

In contrast to the above studies in both hamsters and rats, Honma et al. examined the effects of maternal SCN lesions on

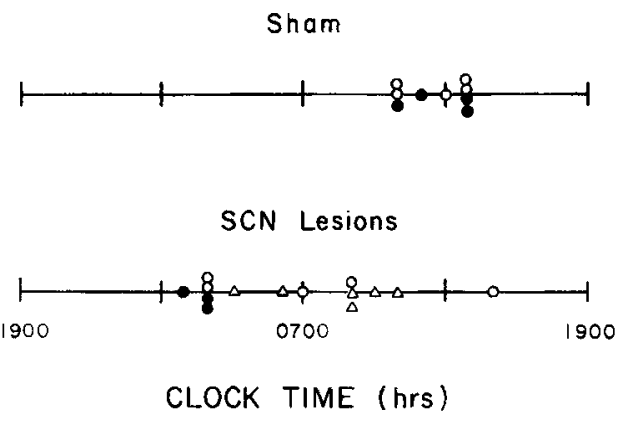

Figure 5. Clock times of drinking offsets at weaning for pups born to sham-operated ( $t o p)$ or SCN-lesioned (bottom) dams. The different symbols represent values from different litters. developing circadian rhythms in rats (Honma et al., 1984a, b) and reported that such lesions on day 10 of gestation did not disrupt the timing of the fetal circadian clock; pups were reared under constant conditions, and the rhythm of plasma corticosterone was monitored in individual pups after 4 weeks of age. These investigators found that the phase of the corticosterone

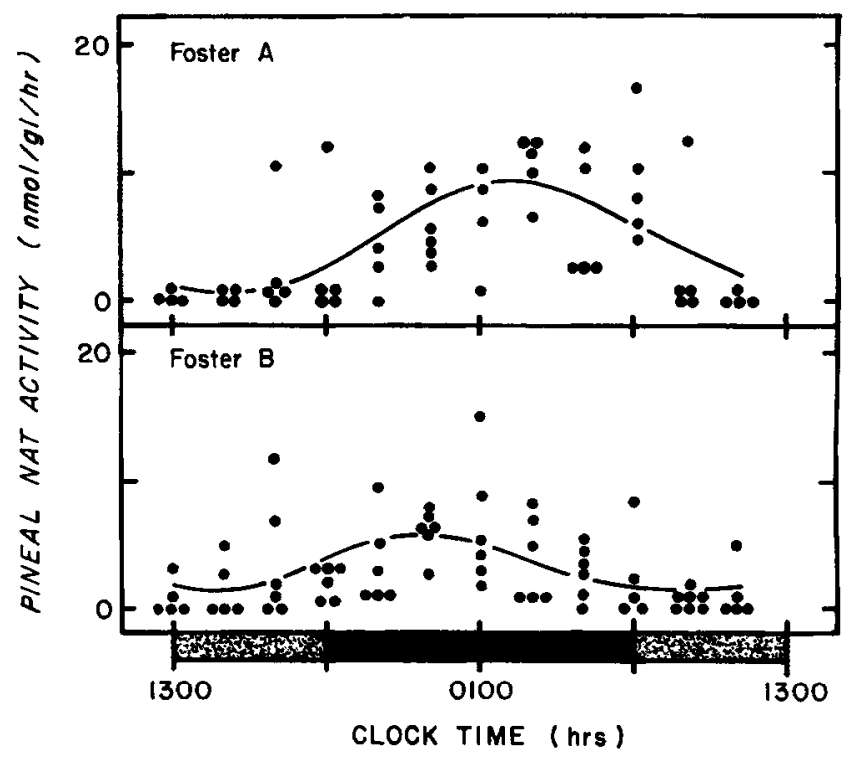

Figure 6. Daily profiles of NAT activity for 10-d-old pups reared from birth by foster mothers. Dams were reared in LD throughout pregnancy, and some received SCN lesions on day 7 of gestation. Two days before birth, the dams were placed in constant darkness. Pups born to intact dams were reared by SCN-lesioned dams (Foster A), and pups born to SCN-lesioned dams were reared by intact dams (Foster B). Pups were killed in constant darkness over a $24 \mathrm{hr}$ period on postnatal day 10 . Each data point is the NAT value of a single pup. Waveforms were derived from sine-wave analysis of the profiles. Stippled area of the lighting cycle represents subjective day. 
rhythm was synchronous among pups born to $\mathrm{SCN}$-lesioned dams but that the phase of these animals was shifted by about $4 \mathrm{hr}$ compared to that of pups born to sham-operated dams. They interpreted this phase difference as showing that the timing of the circadian system in pups from SCN-lesioned dams had already been set by the mother before the lesion and that the phase difference (from the sham group) was due to the longer period of time ( 1 week) that their circadian systems were free running.

While it is possible that entrainment of the fetus by the maternal SCN has occurred by day 10 ( $3 \mathrm{~d}$ after our day 7 lesions), we are concerned with several aspects of the corticosterone studies. First, $50 \%$ of the dams designated as having SCN lesions had only partial damage to the nuclei. It is known that circadian rhythms can persist even though only a small portion of the nuclei remains unlesioned (Robbins and Stephan, 1983). Second, the waveform of the corticosterone rhythm is subject to many external factors, including stress and feeding (Krieger, 1974), and it is unclear precisely what role the SCN play in the generation of this rhythm (Rusak and Zucker, 1979). Third, in all but 1 case, lesioned dams were cesarean-sectioned and the pups were reared by intact foster mothers. Even though in 1 study pups were alternately reared every $12 \mathrm{hr}$ by 2 foster mothers of opposite circadian time so as to eliminate any postnatal maternal influence (Honma et al., 1984b), the intact circadian system of the foster dams could have influenced the timing of the developing rhythm.

The results of our cross-foster studies (experiment 4) show that the maternal SCN are an important component of the mechanism of maternal entrainment during both pre- and postnatal life; pup NAT activity profiles are rhythmic when reared by SCN-lesioned dams either pre- or postnatally but are not rhythmic when reared by SCN-lesioned dams during both the pre- and postnatal periods. Maternal coordination during only the prenatal period (pups born to intact dams and reared by SCN-lesioned dams) is sufficient to maintain relatively normal synchronization among pups. This finding supports our earlier contention that fetal life is a crucial period during which the developing circadian system is coordinated to prevailing lighting conditions by the maternal circadian system (Reppert et al., 1984). The synchronization found with maternal entrainment during only the postnatal period (pups born to SCN-lesioned dams and reared by intact dams) suggests that one must be cautious when using postnatal rhythms to examine prenatal circadian influences.

The results presented here are consistent with the view that the maternal circadian system entrains the fetal clock's endogenous oscillation to prevailing environmental lighting conditions. We are currently investigating the identity of the maternal entraining signal(s). These signals may be different during the pre- and postnatal periods.

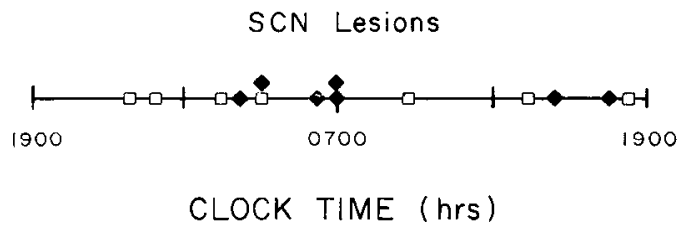

Figure 7. Clock times of drinking offsets at weaning for pups born to $\mathrm{SCN}$-lesioned dams. The different symbols represent values from different litters.
Note added in proof: Since submission of this paper, we have examined the phases of drinking offsets for pups from 2 additional litters born to and reared by SCN-lesioned dams under constant conditions (similar design as Experiment 3). In both litters, drinking offsets for the pups at weaning were scattered throughout the $24 \mathrm{hr}$ day (Fig. 7).

\section{References}

Davis, F. C., and R. Gorski (1983) Entrainment of circadian rhythms in utero: Role of the maternal suprachiasmatic nucleus. Soc. Neurosci. Abstr. 8: 625.

Deguchi, T. (1982) Sympathetic regulation of circadian rhythm of serotonin $\mathrm{N}$-acetyltransferase activity in pineal gland of infant rat. $\mathrm{J}$. Neurochem. 38: 797-802.

Deguchi, T., and J. Axelrod (1972) Sensitive assay for serotonin $\mathrm{N}$-acetyltransferase activity in rat pineal. Anal. Biochem. 50:174 179.

Honma, S., K. Honma, T. Shirakawa, and T. Hiroshige (1984a) Effects of elimination of maternal circadian rhythms during pregnancy on the postnatal development of circadian corticosterone rhythm in blinded infantile rats. Endocrinology 114: 44-50.

Honma, S., K. Honma, T. Shirakawa, and T. Hiroshige (1984b) Maternal phase setting of fetal circadian oscillation underlying the plasma corticosterone rhythm in rats. Endocrinology 114: 1791-1796.

Krieger, D. T. (1974) Food and water restriction shifts corticosterone, temperature, activity and brain amine periodicity. Fndocrinology 95 $1195-1201$.

Moore, R. Y. (1981) The suprachiasmatic nucleus, circadian rhythms, and regulation of brain peptides. In Neurosecretion and Brain Peptides, J. B. Martin, S. Reichlin, and K. Bick, eds., pp. 449-458, Raven, New York.

Moore, R. Y. (1983) Organization and function of a central nervous system circadian oscillator: The suprachiasmatic hypothalamic nucleus. Fed. Proc. 42: 2783-2789.

Partitt, A. G., J. L. Weller, K. K. Saka1, B. H. Marks, and D. C. Klein (1975) Blockade by ouabain or elevated potassium ion concentrations of the adrenergic cyclic $3^{\prime}, 5^{\prime}$-monophosphate-induced stimulation of serotonin N-acetyltransferase. Mol. Pharmacol. 11: 241255.

Pittendrigh, C. S., and S. J. Daan (1976) A functional analysis of circadian pacemakers in nocturnal rodents. I. The stability and lability of spontaneous frequency. J. Comp. Physiol. 106: 223-252.

Reppert, S. M. (1982) Maternal melatonin: A source of melatonin for the immature mammal. In Melatonin Rhythm Generating System, D. C. Klein, ed., pp. 182-192, Karger, Basel.

Reppert, S. M. (1984) The developing circadian timing system: Functional appearance of light-dark entrainment. Pediatr. Res. 17: 154A.

Reppert, S. M. (1985) Maternal entrainment of the developing circadian system. Ann. NY Acad. Sci. 453: 162-169.

Reppert, S. M., and W. J. Schwartz (1983) Maternal coordination of the fetal biological clock in utero. Science 220:969-971.

Reppert, S. M., and W. J. Schwartz (1984) The suprachiasmatic nuclei of the fetal rat: Characterization of a functional circadian clock using ${ }^{14} \mathrm{C}$-labeled deoxyglucose. J. Neurosci. 4: 1677-1682.

Reppert, S. M., R. J. Coleman, H. W. Heath, and J. R. Swedlow (1984) Pineal N-acetyltransferase activity in 10-day-old rats: A paradigm for studying the developing circadian system. Endocrinology 115:918925

Robbins, A., and F. K. Stephan (1983) Circadian rhythms in rats with subtotal lesions of the suprachiasmatic nucleus. Soc. Neurosci. Abstr. 8: 1070 .

Rusak, B., and I. Zucker (1979) Neural regulation of circadian rhythms. Physiol. Rev. 59: 449-526.

Takahashi, K., and T. Deguchi (1983) Entrainment of the circadian rhythms of blinded infant rats by nursing mothers. Physiol. Behav. 31: 373-378. 